is naturaleza 


\section{DOSSIER CRÍTICO SOBRE EL LIBRO:}

\section{LOS SÓTANOS DEL UNIVERSO}

Volumen monográfico de

\section{NATURALEZA Y LIBERTAD}

Revista de estudios interdisciplinares

Número 3

Málaga, 2014

Esta revista es accesible on-line en el siguiente portal: http://grupo.us.es/naturalezayl 


\section{Naturaleza y Libertad}

Revista de estudios interdisciplinares

Número 3, 2014

ISSN: 2254-96682014

Directores: Juan Arana, Universidad de Sevilla; Juan José Padial, Universidad de Málaga; Francisco Rodríguez Valls, Universidad de Sevilla.

Secretaria: Avelina Cecilia Lafuente, Universidad de Sevilla.

Consejo de Redacción: José Luis González Quirós, Universidad Juan Carlos I, Madrid; Francisco Soler, Universität Dortmund / Universidad de Sevilla; Pedro Jesús Teruel. Universidad CEU Cardenal Herrera; Héctor Velázquez, Universidad Panamericana, México.

Adjunto a la redacción: Miguel Palomo, Universidad de Sevilla

Consejo Editorial: Mariano Álvarez, Real Academia de Ciencia Morales y Políticas; Allan Franklin, University of Colorado; Michael Heller, Universidad Pontificia de Cracovia; Manfred Stöcker, Universität Bremen; William Stoeger, University of Arizona.

Consejo Asesor: Rafael Andrés Alemañ Berenguer. Universidad de Alicante; Juan Ramón Álvarez, Universidad de León; Luciano Espinosa, Universidad de Salamanca; Miguel Espinoza, Université de Strasbourg; Juan A. García González, Universidad de Málaga; José Manuel Giménez Amaya, Universidad de Navarra; Karim Gherab Martín, Urbana University, Illinois; Martín López Corredoira, Instituto de Astrofísica de Canarias; Alfredo Marcos, Universidad de Valladolid; Javier Monserrat, Universidad Autónoma de Madrid; Leopoldo Prieto, Colegio Mayor San Pablo, Madrid; Ana Rioja, Universidad Complutense, Madrid. Madrid; José Luis González Recio, Universidad Complutense, Madrid; Javier Serrano, TEC Monterrey (México); Hugo Viciana, Université Paris I; Claudia Vanney, Universidad Austral, Buenos Aires; José Domingo Vilaplana, Huelva.

\section{Redacción y Secretaría:}

Naturaleza y Libertad. Revista de estudios interdisciplinares. Departamento de Filosofía y Lógica. Calle Camilo José Cela s.n. E-41018 Sevilla.

Depósito Legal: MA2112-2012

용 954.55.77.57 Fax: 954.55.16.78. E-mail: jarana@us.es 
Naturaleza y Libertad. Revista de estudios interdisciplinares. Número 3, 2014. ISSN: 2254-9668

\section{ÍNDICE}

PRESENTACIÓN.

DOSSIER CRÍTICO SOBRE EL LIBRO LOS SÓTANOS DEL UNIVERSO

Juan Arana (U. de Sevilla), El problema de la determinación y el diálogo interdisciplinar......11

Miguel Acosta (U. CEU San Pablo, Madrid), La epistemología del riesgo y la

relación ciencia-filosofía. . .25

Carlos del Ama (Madrid), Reflexiones con ocasión del libro Los sótanos del universo ...........57

Luciano Espinosa (U. de Salamanca), De los sótanos al ático del universo ............................93

Gonzalo Génova (U. Carlos III, Madrid), Turing y el rostro del universo ..........................101

Julio A. Gonzalo (U. Autónoma de Madrid), De los sótanos a los confines del universo ....111

Juan Meléndez Sánchez (U. Carlos III, Madrid), De riesgos y metáforas

Andrés Muñoz Machado (Universidad Politécnica de Madrid), Las matemáticas en

Los sótanos del universo

Javier Sánchez Cañizares (U. de Navarra), Sótanos del universo, espejos del alma

Francisco José Soler Gil (U. de Sevilla), Una filosofía de la naturaleza contracorriente y antisistema.

Ignacio Sols (U. Complutense, Madrid), Recuperando la causa formal sin abandonar la causa eficiente. .151

Juan Arana (U. de Sevilla), Los subsótanos del universo. Materiales para un debate. 167 
Naturaleza y Libertad. Revista de estudios interdisciplinares. Número 3, 2014. ISSN: 2254-9668

\title{
RECUPERANDO LA CAUSA FORMAL SIN ABANDONAR LA CAUSA EFICIENTE ${ }^{1}$ \\ Ignacio Sols \\ Universidad Complutense, Madrid
}

\begin{abstract}
Resumen: En la primera parte, celebramos la recuperación de la causa formal en la obra de J. Arana Los sótanos del universo como exigencia cada vez mayor de una actualizada filosofía de la naturaleza. En la segunda parte, urgimos a una defensa de la causalidad eficiente, no desaparecida sino aún más interesante en el actual estado de la ciencia.

Palabras clave: causa formal, causa eficiente, filosofía de la naturaleza

Abstract: First, we celebrate the way J. Arana recovers the formal cause in his work Los sótanos del universo, something increasingly demanded by an updated philosophy of nature. Second, we urge a defense today of the efficient causality, not vanished away but even more relevant in the present state of science.

Keywords: Formal Cause, Philosophy of Nature, Efficient Causality.

Recibido: 16 de marzo de 2013. Aprobado: 12 de diciembre de 2013.
\end{abstract}

Prescindiendo de los aspectos divulgativos — precisamente los más amenos-, trataré aquí el meollo filosófico del reciente libro Los sótanos del universo del filósofo de la naturaleza Juan Arana. Se trata, a mi parecer, de la recuperación de la causalidad formal en contra de las concepciones materialistas ciegas a la forma como principio constitutivo del ser. Estas

$1 \mathrm{~J}$. Arana, Los sótanos del universo. La determinación natural y sus mecanismos ocultos, Madrid, Biblioteca Nueva, 2012. 
concepciones reducen, en particular, la causalidad a mera causalidad eficiente, también ésta blanco de los ataques de la filosofía moderna.

Arana recuerda el carácter problemático del principio de causalidad, entendida ésta como causalidad eficiente. Aunque él divulga sobre modos recientes en que este principio se ha propuesto, permítaseme enunciarlo aquí, para este análisis, del modo más clásico: todo lo que es, pudiendo no ser (es decir, todo lo contingente, por ejemplo todo lo que empieza a ser), es traído al ser, u ontológicamente "producido", por otro u otros seres, su causa eficiente o causas eficientes. Recuerda Arana el ataque de Hume contra este principio al presentar la causalidad como una mera creencia, basada en la costumbre: decimos que $\mathrm{C}$ es causa de un efecto $\mathrm{E}$, significando con ello que hay entre $\mathrm{C}$ y $\mathrm{E}$ una conexión necesaria - de la que no tenemos impresión alguna ni podemos demostrar que la haya-, cuando nos hemos acostumbrado a la contigüidad temporal entre ambas impresiones $\mathrm{C}$ y $\mathrm{E}$.

Juan Arana divulga acerca del intento de salvación de la causalidad como apriorismo del entendimiento humano en la filosofía de Kant, pero también del talón de Aquiles de esta filosofía: el ignotum X causa sus impresiones en nosotros, siendo así que Kant no ha considerado la causalidad algo real sino una categoría mental.

También encuentra que puede presentar inconveniente para el común acuerdo el principio de razón suficiente — versión racional del principio de causalidad - según el cual todo lo que es tiene una razón de ser y de ser como es, es decir, que hay una razón o explicación de todo lo que existe y sucede. Y es que la existencia de una razón suele confundirse con el hecho de que podamos conocerla, y éste a su vez, con que podamos conocerla por los sentidos y la experimentación, lo que lleva a muchos a hablar de azar (contra- 
vención del principio de razón suficiente) cuando sólo se debiera hablarse de azar gnoseológico y epistemológico. Para ser precisos, hay que distinguir entre

1) Azar, es decir azar absoluto u ontológico, entendiendo por tal la ausencia de razón alguna, lo que según el principio de razón suficiente no se da nunca.

2) Azar gnoseológico o ausencia de razón cognoscible por nosotros.

3) Azar epistemológico o ausencia de razón cognoscible por la ciencia experimental.

Esto parece ser lo que lleva a Arana a una versión del principio de causalidad que sólo enunciará al final, pero que aparece ya antes a lo largo del libro como aplicado a las diversas cuestiones sobre causalidad o azar de que trata su obra Los sótanos del universo, a la vez divulgación sobre temas relacionados con la causalidad y ensayo filosófico con propuesta propia sobre la causalidad, esencialmente propuesta de recuperación de la causalidad formal.

Para explicar su propuesta, acude Arana al principio de determinación, que describe así: "este último concepto es extraordinariamente amplio, pero está bastante bien definido: es todo aquello por lo que cualquier ente real existe, en vez de no existir y es de un determinado modo, en vez de ser de otro modo" (he preferido la expresión "en vez de" a su "mejor que", como traducción del "rather" inglés). Este principio, enunciado explícitamente por Nicolai Hartmann, es de tal generalidad que nada se le escapa en ninguno de los ámbitos del ser, por lo que puede ser por todos aceptado como principio universal, aplicable a todos los seres. Pues bien, la idea de Arana es tomarlo como principio de causalidad universal mediante una ampliación del concepto de causa que equipare determinación a causalidad. Para ello sólo tendrá 
que volver al antiguo concepto de causa en Aristóteles, quien hizo bien en no definir lo que en realidad es un concepto primario, y por tanto indefinible, sino limitarse a rescatarlo del lenguaje ordinario en sus cuatro acepciones de causa final (paradójicamente, la primera), causa eficiente, causa material y causa formal. Dicho así, el principio puede sonar tautológico, en el sentido de que nadie, ni Heráclito ni los más materialistas, admitirían que exista algo si se retiran sus causas eficiente, final, material y formal, es decir si no ha sido hecho por nada, ni hecho para nada, ni está hecho de nada, ni es nada. Pero el materialista sólo admitiría causa material, mientras que el principio reclama "todo aquello por lo que existe", dejando claro, de hecho claro a lo largo de todo el libro de Arana, que este "todo aquello" ha de ser realmente "todo", y por tanto incluyente de la forma, como determinante del ser. Recupera así la forma, pero no como lo había hecho Kant, como mera forma a priori, o forma-en-facultad-de-conocer, sino de verdad, como causa formal del ser, como principio constitutivo del ser, como forma-en-la-materia o hile-morfismo.

Esto es una exigencia del actual desarrollo de la ciencia. El pensamiento moderno había olvidado toda causalidad que no fuese eficiente, y cuando la misma causalidad eficiente entró en crisis la reemplazó por necesidad legal (dejó de decirse "los cuerpos caen porque los atrae la tierra" para decir "los cuerpos caen por la ley de la gravedad"). Pero semejante reedición de la forma, para uso de la ciencia, no era reedición como causa formal, forma en la realidad material, sino como forma en nuestro conocimiento científico de ella, versión pues epistemológica de la recuperación de la forma llevada a cabo por Kant en su sistema filosófico. Pero la ciencia actual parece reclamar a gritos la vuelta de la causalidad formal, forma que in-forma ese conglome- 
rado de protones, neutrones y electrones que es la materia, pues sin relación a ella no podemos siquiera hablar de vida. Esta necesidad comenzó a hacerse sentir en la in-formática, donde un reduccionismo (a pura materia) sería, por definición, impensable, pues la in-formación en un programa o software no es reducible a mero hardware. $\mathrm{Y}$ en la actualidad, aparece como protagonista - bajo el nombre de biocomplejidad - en temas de creciente interés que aúnan con la informática el orden descubierto en la transmisión de la vida, al entenderse que el $\mathrm{ADN}$ no es sino un monumental programa informático capaz de reproducirse. ¿De qué manera emerge tal orden a partir del desorden, en dirección contraria al principio entrópico por el que un sistema aislado debe ir del orden al desorden? ¿Es explicable sólo por mutaciones azarosas y selección natural, o debe la ciencia seguir buscando más mecanismos para explicar esta emergencia de la forma?

No es cuestión de contestar ahora a esto, aunque el tema es tratado de modo prolijo en Los sótanos del universo, sino de sólo decir que estas cuestiones claman por la recuperación de la causa formal en filosofía, es decir por el reconocimiento de forma en los seres, y no sólo en nuestro entendimiento, como en filosofía kantiana. No olvidemos que la filosofía aporta al pensamiento humano los conceptos que necesita para su rigurosa articulación, y sin este concepto ni siquiera son planteables los problemas planteados en el contexto de la más reciente ciencia. Éste es el requerimiento de Juan Arana en Los sótanos del universo, obra que viene a ser un viaje fascinante por el mundo de los seres inanimados, luego animados, luego hombres pensantes y luego las computadoras con que éstos intentan emular su propio pensamiento, en un despliegue formidable, fiesta de la forma y 
refutación, por vía de hecho, del materialismo, quizá el más profundo interés de Arana en Los sótanos del universo.

De entre los campos sobre los que divulga Arana a favor de su propuesta filosófica, es el de forma-en-la- vida el que más sugerente me resulta, quizá porque soy lego en la materia. De hecho me llama la atención, en el apartado "lo inerte, lo vivo y lo pensante", la versión que Arana propone del test de Turing por la que un autómata o red neuronal pasaría el test del ser vivo si nace, se nutre, se reproduce y muere en términos que ciertas reglas del juego pongan como inequívocos. Explica Arana en detalle el "juego de la vida" ideado por Conway, consistente en una cuadrícula cuyos cuadrados se encienden o apagan, en función de que estén apagados o encendidos sus cuadrados vecinos, según sólo dos reglas sencillísimas. Por lo visto, hay configuraciones iniciales que dan lugar a formas complicadísimas que se aniquilan unas a otras y parece como si luchasen entre ellas por la existencia, sin que pueda creer quien lo ve que todo se sigue de dos reglas tan sencillas. Esto hace pensar si no será eso lo que ocurre entre verdaderos seres vivos cuya lucha por alimentarse - devorar orden para compensar el propio desgaste entrópico- y así subsistir y aumentar el orden de uno a costa del orden de otros. Hace pensar que quizá pudiera reducirse todo a unas pocas leyes, muy simples, y a unas formas iniciales, que quizá un día conoceremos. La improbabilidad de la vida, de la que Juan Arana recuerda habla Jean Monod en su Azar y Necesidad, el verdadero gordo de la lotería que no queremos darnos cuenta de que nos ha tocado, no nos parece entonces tan improbable. Pero observa Arana que azar y necesidad, en realidad la determinación que no podemos conocer y la que podemos conocer, es lo único que queda 
cuando hemos reducido lo existente a realidad material, la causalidad a causalidad eficiente, y causalidad eficiente a aquella cognoscible por la ciencia.

Este problema, planteado en términos de causalidad formal, sería el de reduccionismo versus emergentismo: el reduccionismo diría que la vida "no es más que" química, y ésta "no es más que física", lo que supondría una reducción de la forma a la materia, mientras que el emergentismo apostaría por la distinción de forma - un científico le llamaría orden (como contrapuesto a entropía) o complejidad-, forma irreductible a pura materia. Arana no se muestra partidario de un reduccionismo ontológico, lo que estaría en contradicción con su apuesta por la recuperación de la causa formal, pero sí de un reduccionismo metodológico, recomendable por razones prácticas: si supongo, por ejemplo, que un proceso vital concreto se reduce a un puro proceso químico, quizá llegue a encontrarlo, pero de modo contrario, no.

Algo parecido dice Arana al hablar de causalidad final, pero recuerda que eso es ya habitual en la ciencia, pues hasta el biólogo más irreductiblemente antifinalista recomienda hablar metodológicamente de finalidad pero a la vez que preceptúa que por finalidad sólo se entienda el resultado de la combinación de azar y necesidad en la teoría de Darwin, o, si ésta se mostrara insuficiente, en las versiones alternativas actualmente propuestas, y de las que Arana aporta una breve noticia.

Por aquel "Amicus Plato sed magis amica veritas" que el propio Arana recuerda en su obra, trato a continuación de algo con lo que no puedo mostrar mi acuerdo. En punto tan delicado para la causalidad como es el colapsamiento de la función de onda en mecánica cuántica, tenemos esta descripción de Arana de lo que los físicos hacen cuando la estudian: "es más o menos lo siguiente: aceptan que los conceptos objetos de discusión [por ejemplo, la 
posición] sí pueden ser atribuidos a los entes que componen el universo (fotones, electrones, átomos, e incluso moléculas, etc...) Pero en vez de determinarlos con precisión eligen moverse en el terreno de lo virtual y contemplan el conjunto de todos los valores posibles de las controvertidas magnitudes, valorando la probabilidad de que el sistema se encuentre en cada uno de ellos según la información disponible”. Estaría de acuerdo si Arana hubiese escrito no en vez de sí, es decir que antes de hacer una experiencia para determinar la posición de una partícula, no es que la partícula si ocupe una posición pero no sepamos cuál es, y por tanto tengamos que recurrir a una distribución de probabilidad de cada posición posible, sino que realmente no ocupa ninguna posición. Esta aleatoreidad, que nos obliga a usar probabilidades, no es el nombre de nuestra ignorancia, sino algo intrínseco de la partícula. Lo que hay es la (densidad de) probabilidad, en cada lugar de que aparezca allí la partícula.

Cierto es que la descripción de Arana ha sido mantenida por muchos físicos, y de hecho algunos físicos aún la mantienen ahora, pero después de que Allan Aspect consiguiera violar experimentalmente las desigualdades de Bell, la física estándar o convencional está por la descripción que dice no, y no está por la que dice sí. Con todo, entre los que aún abogan por la posibilidad del sí hay algún físico de talla excepcional como t'Hooft, para quien el no sería debido a las matemáticas utilizadas hasta ahora para formalizar la situación, pero las matemáticas definitivas podrían ser otras. Sin embargo, lo aceptado por la comunidad científica — después de la refutación experimental de Allan Aspect- es que se trata de uno de los aspectos irrenunciables de la Mecánica Cuántica, es decir aspecto que cualquier teoría ulterior, como la teoría unificada si alguna vez llega, deberá respetar y recuperar como límite clásico (así 158 
como la física newtoniana es recuperada por la relatividad especial como límite clásico al tomar la velocidad de la luz como infinito, y por la mecánica cuántica al tomar la constante de Planck igual a cero)

Y siguiendo en el "magis amica veritas", quisiera mencionar que el lector de la obra de Arana podría quedar con la idea de que los físicos ven indeterminismo en el colapsamiento de la función de onda solamente por la discontinuidad que este colapsamiento supone, en el momento de la observación, para la evolución temporal de la función de onda, evolución que es, en cualquier otro instante determinista. $\mathrm{El}$ indeterminismo en el instante de la discontinuidad no es sólo por el hecho de la discontinuidad sino porque después de ese instante hay muchas opciones posibles a seguir - muchos lugares posibles donde puede aparecer la partícula - cada uno con una cierta (densidad de) probabilidad. Si después del instante de discontinuidad sólo hubiera una posibilidad — como en el gráfico de una función discontinua, especie de camino único pero dislocado por un antiguo terremoto- la situación sería aún determinista. Sin embargo no hay determinismo en el fluir por un camino, aunque ningún terremoto haya roto su continuidad, si se llega a un punto en que el camino se bifurca, pues el futuro ofrece entonces varias posibilidades.

Por eso hay ya indeterminismo en la mecánica clásica, aunque esto aún no se comprendía en el tiempo del famoso discurso determinista de Laplace, que trajo de cabeza a los pobres ingenuos que en su época creían, por razones filosóficas, en la libertad (como se ve, los tiempos se parecen). En efecto, así sucede en los problemas de movimiento - como el de tres cuerpos, sol, tierra, luna- que están descritos por una ecuación que admite como solución una curva - por supuesto continua - que en un punto no sea diferenciable, 
lo que llamamos punto singular: supongamos, para comprenderlo, que la solución es una uve, fluyendo el tiempo hacia arriba, y que el móvil está ahora en el punto en que la uve no es diferenciable, es decir donde presenta un pico, o sea supongamos que el móvil está ahora en el vértice de la uve. ¿Dónde estará dentro de unos instantes? ¡Está indeterminado por cuál de los dos brazos de la uve debe fluir!

Recientemente se ha puesto el acento en la imposibilidad de predicción del futuro que suponen ambos indeterminismos — clásico y cuántico- combinados: para un movimiento descrito por una ecuación diferencial que admite como solución una curva con una singularidad, cuanto más cerca estemos de la singularidad con más precisión necesitamos conocer los parámetros de posición e impulso (producto de masa por velocidad) para poder predecir su comportamiento futuro. Por tanto, si ese móvil es una partícula, o un condensado material que se comporte como una sola partícula - lo que en la actualidad es posible-, como el principio de indeterminación de Heisenberg impide que haya precisión ilimitada simultáneamente en posición e impulso, su futuro es intrínsecamente impredecible. Esto no es ciencia ficción: del satélite Hiperion de Saturno, se ha calculado que es intrínsecamente impredecible su comportamiento de aquí a más de veinte años. Esta existencia de indeterminación, clásica, cuántica, o combinada, no es un argumento a favor de la posibilidad de voluntad libre, pues es indeterminación para la ciencia y la voluntad libre es un tema filosófico, concepto sin sentido para la ciencia y para su método. Pero ha removido el obstáculo que según Laplace ponía la ciencia a la posibilidad misma de libertad —antes del descubrimiento de estos indeterminismos- pues lo que la materia deja indeterminado, bien puede ser autodeterminado por nuestra voluntad. 
Volvamos ahora, en el contexto de estas indeterminaciones aparecidas en ciencia, al principio de causalidad, en su versión de causalidad eficiente. Puede parecer que abogar por el sí en la interpretación del colapsamiento de la función de onda antes recordado, o sea abogar por la aleatoriedad intrínseca en la cuestión que hemos tratado, suponga abogar por una violación de este principio. Pero fijémonos que el principio no trata de causa eficiente que podamos conocer, y menos que sea cognoscible por la ciencia experimental. ( $\mathrm{Si}$ yo me encuentro con un montón de letras y veo que una detrás de otra parecen decir algo que tiene sentido, y de hecho con tanto sentido que resulta una obra maravillosa, por ejemplo el Quijote, yo sé que esas letras han sido conjuntadas por una mente pensante y no con un bombo de la lotería, una a una, al puro azar, y lo sé aunque no me lo diga ni la biología, ni la química, ni la física, ni las matemáticas. Y es que el conocimiento, y en particular el conocimiento por causas eficientes, no se agota en lo que pueda yo conocer por mis cincos sentidos o por esa prolongación de los sentidos que son las ciencias experimentales. Sé que aquello lo ha escrito una mente pensante, pero en modo alguno pretenderé que sea éste un conocimiento científico). Pues bien, en el caso del colapsamiento de la función de ondas, el principio de causalidad eficiente no es en modo alguno violado, puesto que hay causa y además causa que podemos conocer por la ciencia experimental: la causa del colapsamiento de la función de onda es la observación misma, es decir esa interacción entre el sistema macroscópico que es el observador o su laboratorio y el sistema microscópico que es la partícula. Sólo sería violado el principio de racionalidad que afirma que hay una razón o explicación de todo lo que sucede, si es que este principio afirmase también que esa explicación deba ser en ciencia experimental, puesto que no hay ni habrá explicación en 
ciencia experimental alguna de por qué la partícula ha aparecido en un lugar en vez de otro, siendo ambos probables. Por tanto, si a pesar de todo insistimos, por razones filosóficas, en que el principio de racionalidad debe cumplirse, si insistimos en que nada puede suceder que no tenga explicación, entonces nos vemos forzados a admitir que hay explicación sobre la realidad más allá de la meramente científica, y por tanto forzados a admitir más realidad que la mera realidad material o realidad alcanzable por los sentidos o su prolongación en las ciencias experimentales. Pero no pretenderé que esa afirmación sea científica, sino filosófica, pues a ella llego por un principio filosófico ${ }^{2}$.

2 En el caso de un colapsamiento de la función de onda causado por un dispositivo de acción aleatoria, por ejemplo dependiente de una emisión radioactiva (como es el dispositivo que mataría al gato de Schrödinger), la radioactividad del elemento elegido para el dispositivo sería la causa eficiente. Pero algunos, como Stuart A. Kauffman, prefieren hablar de acausalidad eficiente, debido a su carácter aleatorio. Pero vendríamos con esto a lo mismo, pues sería acausalidad física eficiente, por lo que, si mantenemos el principio filosófico de causalidad eficiente, venimos a realidad que no nos da la mera ciencia de la naturaleza. La física ha dejado espacio libre a la realidad filosófica de la voluntad libre y responsable, la cual, por no ser realidad física, no nos presentará nunca la ciencia. El reciente estudio de Kauffman "Five problems in the philosophy of mind" es especialmente interesante porque divulga sobre las actuales sugerencias de fenómenos cuánticos en el nivel celular y por tanto en el funcionamiento del cerebro, lo que llevaría esta discusión precisamente allí donde se generan nuestras decisiones. Y también por que rechaza el puro cálculo de probabilidades en la evolución, en la medida que no son calculables los casos posibles, es decir las mutaciones preadaptables (es decir aquellas que seguidas de otras mutaciones darán un carácter adaptable al ambiente). En ese sentido, dice Kauffman que la evolución es también, como nuestra mente, "lawless" pero no "random", es decir que no sigue un comportamiento determinable por 
De hecho, no sólo la ciencia moderna no ha presentado ningún caso de violación del principio de causalidad eficiente, sino que ha echado por tierra todos los argumentos por los que Hume desterró la causalidad eficiente de la filosofía. Recordemos, que Hume retaba al lector a encontrar un solo razonamiento, que ni existe ni existirá jamás, por el que un ennegrecimiento del papel se haya de seguir necesariamente del brillo y calor al que llamamos fuego. Obviamente, no es posible encontrar una conexión necesaria entre las impresiones de brillo y calor y la de ennegrecimiento del papel, y el problema está en que Hume sólo admite impresiones, no algo de lo cual sean brillo y calor las impresiones que deja en nosotros. Pero la ciencia moderna ha entendido que son propiedades ambas físicas de un átomo, y átomo del cual se han encontrado otras propiedades —las propiedades químicas dependientes del número de electrones en la última órbita- de las cuales puede deducir necesariamente la reacción que captamos como carbonización del papel. Son éstas propiedades químicas que a su vez se siguen de propiedades de la estructura del átomo, deducibles éstas de los principios de la Mecánica Cuántica. Lo mismo puede decirse de cada uno de los otros ejemplos que pone Hume, como que nunca se podrá comprender por qué el pan nos alimenta, etcétera.

la ciencia pero tampoco es mera aleatoreidad. Y añade que, por esta razón, ambas se resistirían a una teoría física final (Theory of everything) en el sentido fuerte de que con sus leyes pudiese ser determinada toda realidad, incluido el conjunto de la evolución y nuestro comportamiento. Kauffman observa que queda pues espacio para una voluntad responsable, con lo que viene a expresar lo que yo he llamado voluntad libre (para él aún podría llamarse voluntad libre a una voluntad irresponsable que generase sus decisiones por puro azar, lo cual ha descartado pueda ser el caso). 
Sin embargo, creo que no hemos tenido que esperar a la ciencia actual para refutar a Hume, y que el argumento al que retaba se podría haber aportado ya en su época, aunque inspirado en la epistemología actual: Afirma Poincaré en La Science et l'Hypothese que el conocimiento científico se basa en dos hipótesis: Primera, la hipótesis de simplicidad, según la cual el conocimiento busca siempre la solución más simple (él se refiere al conocimiento científico, pero vale en general). Es hipótesis, ciertamente, pero la más natural para el conocimiento, pues sin la suposición de que hay unidad y armonía en el universo - Arana habla de ella repetidamente en su libro-y de que entender la naturaleza es llegar a captar esa unidad - lo que hacemos cuando con algo muy simple explicamos mucho- no hay en realidad conocimiento sino ver el mundo como un mosaico de seres y hechos solipsistas: nada que explicar, sólo mirar. Para ilustrar esta idea, recuerda Poincaré que al comprobar Kepler que todas las posiciones observadas de Marte estaban en una elipse, concluyó que se mueve en esa elipse, aunque muchas otras curvas pasen también por las posiciones observadas, pero la elipse es la más simple de todas (la de grado más bajo, grado dos). Y, segundo, la bipótesis de regularidad según la cual la naturaleza responde siempre, bajo las mismas condiciones, del mismo modo (modo que puede ser aleatorio, como en las observaciones cuánticas). De hecho, creo que ésta es consecuencia de la hipótesis anterior porque la explicación más simple de que hasta ahora haya respondido siempre de un determinado modo es que necesariamente tenía que ser así, desechando como rebuscada la explicación de que haya sido por un cúmulo de casualidades. Ergo, así debe suceder también en el futuro.

Aplicando la hipótesis de simplicidad que vale no sólo para el conocimiento científico sino para el conocimiento mismo, pues es la descripción 
misma de lo que hacemos al intentar entender algo - buscar la explicación más sencilla, de modo que poco explique mucho-, podemos aportar sin problema el razonamiento que pide Hume: La explicación más simple de que el fuego haya carbonizado siempre el papel no es un cúmulo increíble de coincidencias, sino que existe un nexo necesario, pero aún desconocido, entre propiedades quizá aún no conocidas del fuego, y el cambio de color que hasta ahora se ha experimentado en un papel cercano. En consecuencia, deberá seguir siendo así en el futuro ${ }^{3}$. Y lo mismo podemos decir de todas las ocurrencias de la causalidad eficiente: ¿cómo sé por ejemplo que aquellas letras que compusieron el Quijote no habían sido fortuitamente conjuntadas sino ordenadas por una mente y de hecho una mente ingeniosa? He desechado la explicación de que hubiesen quedado así al azar por increíblemente rebuscada, y he acudido a la explicación más sencilla, explicación por supuesto no científica, pero sí verdadero saber. Es de este modo, ni biológico ni químico ni físico ni matemático, que tenemos conocimiento de la voluntad libre de los demás como experimentamos la propia nuestra, conocimiento

3 Se objetará, ya en términos actuales, que la razón que aporta la ciencia se basa en propiedades químicas, reducibles a la postre a Mecánica Cuántica, y que ésta se basa, como toda teoría física, en unos postulados que son leyes universales y que no pueden por tanto tener justificación experimental, ya que lo particular no puede justificar nunca lo universal. Creo que puede justificarlo si se añade la hipótesis de regularidad, reducible como hemos visto a esa hipótesis de simplicidad, tan natural que es la descripción misma de nuestro entender. Queda con todo el carácter hipotético, acrecentado por el hecho de que los postulados no suelen ser leyes directamente experimentales sino más bien compatibles con ellas. Sin embargo, estimo que esto viene asumido en la exigencia misma de nexo entre causa y efecto en términos de la ciencia actual, ya que toda teoría científica es, por su propio método, hipotética. 
que aunque no es científico, la ciencia actual ha dejado como posibilidad, tal como hemos visto. (Compton, quien como creyente se sentía incómodo con cualquier planteamiento que negara la libertad humana, ponía su dedo delante de sus ojos y lo movía diciendo que si una teoría física afirmase que no lo movía al arbitrio, él antes creería a su dedo más que a esa teoría). Es ése también el modo como los hombres llegamos a tener un conocimiento de Dios — no científico pero sí filosófico- como causa del mundo, no sólo causa de su ser sino también de su orden, al tiempo que tendremos razón en expulsar de la ciencia el diseño inteligente, pues la ciencia es autónoma y por tanto "no necesita esa hipótesis". Si ese orden lo ha dejado el Creador en leyes muy simples, que estudia la ciencia, tanto más admirable es por ello, como dice Darwin al final del Origen de las Especies:

Hay una grandiosidad en esta visión de la vida, con sus diversos poderes, habiendo recibido el aliento original del Creador (having been originally breathed by the Creator) en unos pocas formas o en una sola; y en que, mientras este planeta rodaba siguiendo la ley fija de la gravedad, hayan evolucionado y estén evolucionando desde un comienzo tan simple un sin fin de formas bellísimas y maravillosas.

Ignacio Sols Lucia isols@mat.ucm.es 